\title{
An Integrated Signal Allocation Model with Effective Collision Resolution Model for Performance Enhancement of Wireless Sensor Networks
}

\author{
Aswani Lalitha ${ }^{1 *}$, Gangireddy Harinatha Reddy ${ }^{2}$ \\ ${ }^{1}$ Jawaharlal Nehru Technological University Anantapur, Ananthapuramu 515002, Andhra Pradesh, India \\ ${ }^{2}$ Department of ECE, NBKR Institute of Science \& Technology, Vidyanagar, SPSR Nellore 524413, Andhra Pradesh, India
}

Corresponding Author Email: aswanilalitha24@gmail.com

https://doi.org/10.18280/ts.380512

Received: 3 August 2021

Accepted: 29 September 2021

\section{Keywords:}

signal allocation, collision reduction, performance enhancement, integrated model, labelled weighted model

\begin{abstract}
A Wireless Sensor Network (WSN) differs from conventional wireless or wired networks in that it interacts with the environment. Orthogonal Frequency Division Multiplexing (OFDM) was investigated as a possible interface technology for making effective use of bandwidth. Such networks have been proposed for a variety of purposes such as search and rescue, disaster assistance, and smart positioning systems. These applications often require a large number of wireless sensors that are powered by batteries and are designed for longterm, human-free deployment. Collisions between network nodes can significantly degrade performance in WSNs. Although increased bandwidth facilitates wireless access to high data frequencies, it is prohibitively expensive to increase due to spectrum limits. This necessitates making good use of the available bandwidth. OFDM has been considered as a possible interface mechanism for efficiently utilising bandwidth. While many signals available in WSN technology can be employed to mitigate collisions, multi-signal allocations may have a significant impact on the efficiency of multistage communications. Real-time multimedia flow raises the chance of sensor network failures and congestion, which reduces the efficiency of Quality of Service (QoS). The main goal of the Signal Allocation Scheme is to allocate an appropriate number of signals to any node in order to use professional bandwidth and assure QoS. Load balancing is intended to measure and prevent collisions caused by the number of available slots in the frame. Preparation is another important component in preventing collisions because it decreases delay and optimises energy utilisation. In this paper, an Integrated Signal Allocation Model with Effective Collision Resolution Model (ICAM-ECR) is used to deploy non-overlapping signals dynamically for varying application loads based on expected bandwidth estimation. The suggested model is compared to standard methods, and the findings reveal that the proposed model outperforms existing models.
\end{abstract}

\section{INTRODUCTION}

Small and affordable strategies that include a microcontroller with low power, multiple sensors and a communications radio consist of WSNs. They are independent ad-hoc networks which can sensor, process data and transmit various physical parameters to a user through the use of multihop communications [1]. They offer a versatile, independent, low-cost solution, particularly for places with limited accessibility, for a variety of distributed monitoring applications [2]. Given the difficulty of replacing batteries, WSNs must use efficient energy storage schemes to save energy for longer life. The use of renewable energies, such as sunlight, vibration, heat etc. for long-term operations at WSNs is an alternative solution [3].

In WSN, collision resolution is a key concern. There are disadvantages in terms of energy consumption and latency method in current collision resolution approaches [4]. If several nodes intend to concurrently send packets to the common medium, a collision occurs [5]. Unforeseen collisions harm the use of wireless signals by wasting resources and degradation of network performance. low-duty cycles are used by most WSNs as energy is reduced [6]. In order to save energy, sensor nodes try to stop their radio, which squeezes the time available for packet transmits between nodes and may lead to collisions. For event detection, for example, WSNs add a wider risk of collisions to predictable busy traffic triggered by events [7].

There have been suggestions for dealing with WSN collisions on various protocols of MAC (Medium Access Control). In principle, all of these ideas adopt the CSMA/CA mechanism. A node senses the signal in case of a collision before transmission and retrieving it randomly [8]. The main principle underlying CSMA/CA is that a station ought to be able to obtain while broadcasting in order to identify a collision between two stations. In wired networks, if a collision happens, the energy of the received signal nearly doubles, and the station detects the probability of a collision. CSMA/CA is a networking multiple access strategy wherein the nodes seek to prevent collisions by initiating transmission only once the channel is felt to be "idle."

The duration of the random back-off window is raised exponentially to prevent secondary collision [9]. The investigation demonstrates that in general wireless networks, CSMA-based techniques can produce acceptable outcomes. However, the efficiency of duty cycling WSNs may decline 
significantly, particularly when the network operates with uncoordinated burst traffic [10]. Node coordination is an effective method for avoiding recurring collisions [11]. RIMAC, for example, is a WSN MAC protocol initiated by the receiver. If there is a collision, the recipient requests several senders with an exponentially increasing back off window [12]. However, this results in significant lag in distribution and energy use. As a result, the process of identifying and resolving collisions takes time but consumes no energy because only one sender is located and access to the signal is gained in each round [13]. Control packets must also be longer than usual to resolve collisions and can conflict with other packet transmissions that restrict network output further. The Network Architecture is shown in Figure 1.

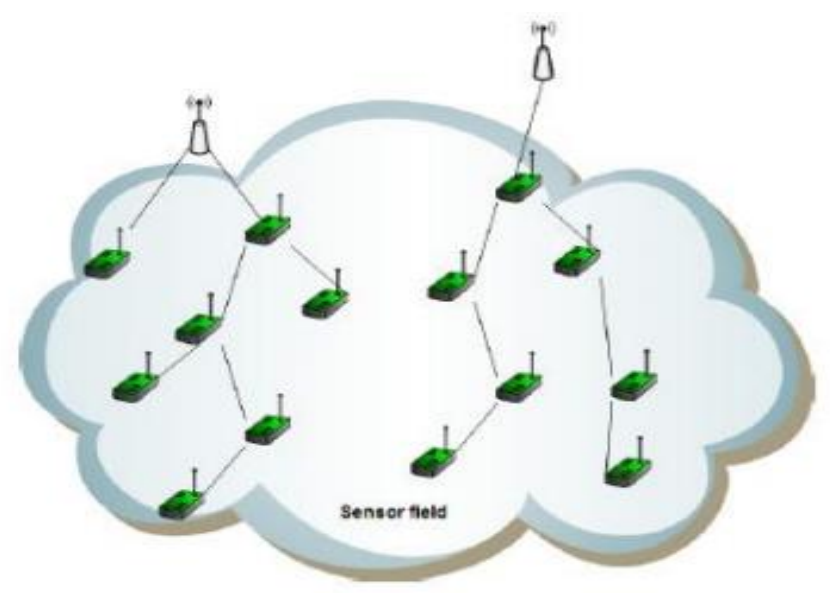

Figure 1. Network architecture

Self-organization has been one of the most significant distributed strategies in recent years [14]. This method allows a sensor network to produce emergent behaviour in which nodes communicate independently and collaborate autonomously. The goal is to complete tasks that are beyond the capacity of a single node [15]. Protocols designed for dispersed sensor network must be able to provide optimal energy usage while taking into account mobile nodes, external noise, limited batteries, and message loss, among other factors [16].

In communication systems, wireless networking plays an important role. Modern wireless multimedia applications with restricted spectrum resources need a greater rate of data and higher bandwidth for multiple users [17]. In order to satisfy the portability requirements of future wireless systems, low energy use and smaller Silicone areas are crucial. The energyefficient implementation and cost-efficiency of advanced communication algorithms are still the design challenge, requiring optimisation of wireless device design; system/algorithm, architecture and technology deployment [18].

New developments have proven or demonstrated unparalleled wireless spectral efficiency in knowledge theoretical and communication algorithms [19]. However, many of them need very high processing power and have therefore not been able to incorporate data rates in real time and using traditional devices with acceptable energy consumption and expenses for typical portable applications requiring wireless communication [20]. The distinctive features of this application domain can be exploited in order to reduce the cost of implementation. The focus of the work is on providing the wireless networks, especially wireless sensor networks with energy efficient and collision prevention protocols [21].

The networking group has made many efforts to develop routing protocols for energy saving in one-signal sensor networks [22]. Regrettably, any node suffers from overheard transmissions from all other nodes in its scope, resulting in high energy wastage when only one signal is used [23]. This can be alleviated by the use of several network signals. Using multiple signals also helps to reduce interference and increase communication efficiency in the network [24]. Current WSN hardware, like MICAz and Telos, that use the radio CC2420, provide multi-signal systems (16 signals with $5 \mathrm{MHz}$ of intermediate frequency spacing). Problems imposed by energy supply fluctuations can be solved using methods to adjust their energy consumption dynamically on the basis of projected energy supplies. It is proved that selection and routing of complex signals can solve this issue [25]. The design of successful frameworks for jointly selecting energy management signals and routes is therefore a complex problem since it is necessary to resolve wide-ranging network adaptations, rather than individual node modifications.

Collisions on wireless systems can generally be a key cause of amplified latency and retransmission of packets. As energyconstrained wireless networks collide, additional latency and retransmissions are equivalent to excess power consumption. The energy on the remote platform is premium [26], so collision prevention will improve the network's life span. Current network sensor solutions attempt either with the help of TDMA or RTS/CTS to solve the collision problem in the MAC layer, but applications-specific information can't easily be exploited by MAC layer solutions [27] or they try. The collision in the network is indicated in Figure 2.

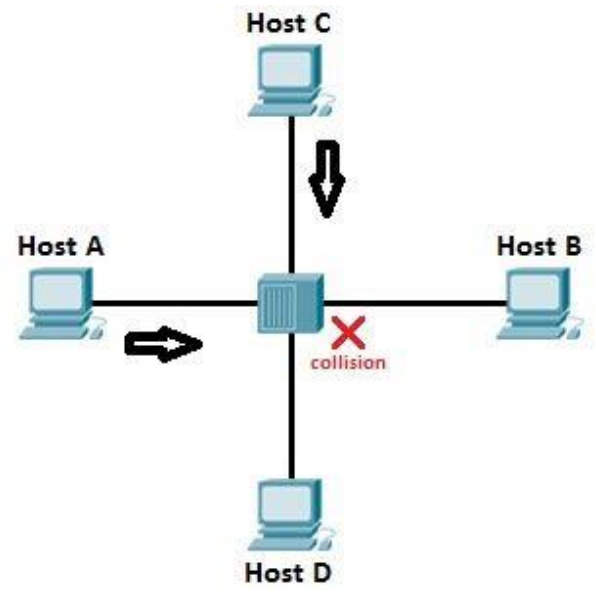

Figure 2. Collision model

When two or even more nodes attempt to send a packet all over the network at the same time, they collide. Typically, a node in a WSN does not know when to wake up in order to receive a data packet, thus it must maintain its radio on, which consumes the majority of the energy. A collision avoidance mechanism using domain-specific information will complement and increase the efficiency of the MAC layer, given a specific type of application [28]. When implementing a collision prevention system, wireless communication can be partitioned into two types: a single and many packages. Previous packet arrivals don't have information on potential arrivals for a single packet communication; arrivals are basically random. The presence of a 'next' packet is specified in multi packet communication. A correlation now exists 
between past and future arrivals. In this paper, an Integrated Signal Allocation Model with Effective Collision Resolution Model in Routing for Performance Enhancement of Wireless Sensor Networks is proposed that improves the performance of the system.

\section{LITERATURE SURVEY}

Sensor networks are well-researched for Tree-based routing. Lei et al. [1] proposed Collection Tree Protocol (CTP) available in Tinyos 2.x are two very common tree-based systems. These tree-based protocols for selection are designed to make best possible communication between any of the datagrams in one of the network's root nodes. Some of the nodes begin with the root nodes or sink nodes to advertise themselves. The other nodes are connected to the collection tree using root advertisements. If any physical parameter is collected by a node, the tree shall be sent. Because the network can contain many root nodes, the data are provided at a minimal cost. The protocols are free of addresses, so that a packet is not sent to a specific node, and is based on routing costs. Recent times have been very attentive to multi-signal routing in wireless networks. Most of the work reported in this field either takes on a multi-radio transceiver at each node or generates high signal negotiation control overheads. These systems are not suited for WSNs, where a single radio transceiver normally is used on each sensor. Furthermore, overhead should be reduced because energy resources are premium.

A centralised strategy to pick hop equipment was proposed by Roy et al. [2]. When the sensor node is hybrid, entirely wireless but much less movable, or fully mobile with a known sensor position, centralised cluster generation can be used. Sensors within a group are only meant to communicate with the cluster head in a cluster-based inter process communication. The cluster heads collect and process sensor readings from the clusters, as well as maintain the communication with the access point. The clustering is motivated by the desire to reduce energy consumption across all sensors. To select the hop unit, the signal state data is used. The central system selects a range of hop devices to help the end-to-finish bit error rate by using signal statistics and decision strategy. The option of a number of hop devices with binary symmetrical signals is the upper boundary. Similarly, Sharma and Bhondekar [3] introduced the source-to-relay energy-minimization model. The model considers that the canal state information is compressed and the subset of bits only is passed to a hop unit that is considered to be good bits. They also followed a centralised approach to select a relay system that meets the Bit error rate and the Packet Rate for selecting thresholds. Simulation results show improvements in performance for equal compression, energy consumption and bit error rates.

Data packets corrupted lead to retransmissions. Packet transmissions are amongst the most energy intensive operations in resources-constrained wireless sensor networks. Thus, reducing collisions is one efficient way of reducing energy consumption. The slowdown of packet transmissions will minimise collisions; but this will also prolong the completion time for code dissemination. Radio listening time also entails a non-denial cost, even though not as high as energy consumption like packet transmissions, unless an SMAC energy conscious MAC is used in special instances.
Kaur and Kumar [4] proposed a strategy for optimising the lifetime of the sensor network. Taking into account both the recognition and the data packet, transceiver power is optimised Choosing the high power node that reduces the loss of the handshake. It does not, however, ensure maximum network life. The linked-level handshaking mechanism to reduce power consumption was presented here. The simulation is carried out to evaluate the efficiency for different network sizes and density.

Emad and Ion [7] introduced an energy-efficient sensor network routing strategy by clustering technique. They implemented a clustering protocol based on hierarchy. Their model reduced the dissipation of energy and the time for reclusion. As a routing parameter, the sensor interface throughput is used. The packet transmission is achieved by multihop or direct method based on the throughput standard. In comparison to the LEACH Protocol, simulation is done taking into account mobility of sensor device which shows significant improvements in network life, energy efficiency and performance.

Protocols are algorithms distributed which enable multiple users to communicate (machines or nodes). These algorithms, however, are very difficult to understand and implement. It is therefore essential to ensure that these protocols are developed and implemented in order to better make use of them. Perhaps more critical is the question of the design and implementation of protocols in wireless sensor networks as sensor node positions can alter and collide. Collisions are caused by nodes that meanwhile transmit information through a medium of transmission. Thereafter efforts to lower or restrict collisions are undertaken in the MAC layer. One typical example is the LMAC protocol, which is modelled and evaluated using a time-consuming model checker. The exploratory effects of different TDMAs are now being presented by Tanwar et al. [10]. MAC agreements using the OMNeT++ discrete evaluation system. Furthermore, the author considers the test consequences for wireless sensor systems for SMAC, EMAC and LMAC conventions.

\section{PROPOSED MODEL}

The use of Integrated Labelling system is a simple method for reducing collisions. Time is divided into vast eras, where each source selects a time to submit by random measure. Since the time is large enough relative to the time of sending packet, the likelihood of conflicting sending times for some other source is small. Per source can only send one packet per time by definition. Latency is also directly proportional to the size of the time. Ideally, the risk of potential collisions can be reduced without increasing the latency or number of retransmissions substantially [29].

Avoidance of congestion on a network for packet switching is equivalent to a collision in a wireless network. In each case, in presence of an anomaly, the source throws the data rate. The effusive rate would influence the data packets arrive and therefore avoid repeated collisions due to overlap periods in the prevention of collisions. By slowing down the source, large traffic on the receiver could also dissipate. This latter result implicitly implies that all traffic in sources is finite and that the background sources will finally stop after some time. The specification implicitly assumes that a packet loss is a result of congestion in the case of the TCP congestion control. The data from collisions or a link failure may be lost on the user This 
presumption cannot be found in wireless systems.

If we suppose that all sources send packets with the same rate of $1 / \mathrm{T}$ - that is, every active source sends a packet during time frame $\mathrm{T}$, then the recipient can also track packet arrivals and detect significant periods of inactivity within a certain time frame. If a packet loss is caused by a collision, the recipient will send the "largest silent time" information to their primary source. At that time, the source could try to transmit to minimise the likelihood of further crises. The source basically tries to interlink its transmissions with background transmissions while still maintaining a steady rate of data. This technique is known as the avoidance of phase offset collisions using integrated signal allocation model that reduces the collisions.

Colliding on the recipient is not as simple as testing for corrupt packages since both connection losses and collisions can lead to corruption. We can't always inspect the packet to decide its source because it's corrupt. The only thing that can be deduced is that there was a failure. Ideally, when each source packets arrived, the recipient would know exactly. If several packages arrived simultaneously, the receiver could decide whether a collision happened and notify the source of the collision. By inserting a little determinism into the protocol, the optimal solution can be estimated. Since packets may come within the range at any time, a collision cannot occur even when ranges are overlapping. The model for collision detection cannot therefore be used to forecast possible collisions accurately. In accordance with this, if the collision occurred by the end of the scheduled arrival date, along with the transfer shift, a missing packet is defined as collisioninduced loss.

The Integrated signal allocation model verifies the available signals in full length and selects the best signal and allocates for completing the data transmission. The signal strength is estimated as

$$
\begin{aligned}
\text { Strength }_{C H} & =\left(\frac{L i}{I}-1\right) * S i+* D L_{A} \\
& +g * E_{s y n c}+\varepsilon f_{s}
\end{aligned}
$$

Here $\mathrm{Li}$ is the limit of the signals range, DL is the Data loss limit of signal transmitted by antenna $\mathrm{A}, \mathrm{Si}$ is the signal range, $E_{s y n c}$ is the synchronization levels of the signal, $\varepsilon f$ is the region range of the nodes. The squared signal distance level is calculated as

$$
E\left[d_{t o C H}^{2}\right]=\iint\left((m-x)^{2}+(n-y)^{2}\right) p(x, y) d x d y
$$

Here $m$ and $n$ are arbitrary signal region in the limit, $x$ and $y$ are base station location coordinates.

The estimation of Signal Frequency Sf and Time Interval gap Tg can be obtained by jointly maximizing over Sf and Tg. The operation is performed as

$$
C h_{\text {freq }}(S f, T g)=\frac{1}{D_{R} \times L} \sum_{i=1}^{L}\left\|m(I)-x^{*} y(I)\right\|^{T}
$$

Here $\mathrm{D}$ is the squared signal distance, $\mathrm{T}$ is the Thresholds range and $\mathrm{L}$ is limit of the signals to be allocated.

The estimate of best signal data collected from the estimated signals without loss is performed as

$$
\begin{gathered}
D c(m, n)=\arg \left\{\min _{x, y} L_{c h}(x, y)\right\} \\
=\iint \frac{(m-x)^{2}+(n-y)^{2}}{\operatorname{range}(A)+T h} d x d y
\end{gathered}
$$

The non-overlapping signal frequencies are calculated as

$$
C h_{f}=\frac{\sqrt{N L}}{\sqrt{2 \pi}} \sqrt{\frac{\varepsilon f_{s}}{\varepsilon_{m n}}} \frac{L}{d_{t o C H}^{2}} .
$$

Here

$$
\begin{gathered}
\varepsilon_{m n}=\frac{N L_{x y}\left(N_{s r c}-B_{m}\right)+\ell_{t}\left(2^{2 W}-1\right)}{\ell_{j}\left(N L_{x y}+N_{y x}\right)+T h} \\
+\sum_{i=1}^{L}\|m(I)-x * y(I)\|^{T} \\
B_{m}=\left\{\begin{array}{cc}
\frac{\left(2^{2 W}-1\right)}{N L_{x m}}, \quad & \left(2^{2 W}-1\right) \\
\tilde{P}_{m}, & \frac{\left(2^{2 W}-1\right)}{N L m x} \\
N_{z a} & \left(2^{2 W}-1\right) \\
\frac{N L_{n y}}{N}, & B_{m}<\frac{\left(2^{2 W}-1\right)}{N L_{n y}}
\end{array}\right.
\end{gathered}
$$

Here NL is the noise values, $2 \mathrm{~W}$ is the bandwidth limit, Th is the threshold, $\mathrm{m}, \mathrm{n}$ are arbitrary signal region in the limit, $\mathrm{x}$ and $y$ are base station location coordinates. The collisions in the network are identified as

$$
=\frac{1}{N L} \frac{\sum_{n=1}^{N} \min \left\{C h_{\text {freq }}\left\{\left(\varepsilon_{n}^{L}\right)^{M} V_{n}^{L} \psi_{n}^{T h}\right\}\right\}}{\sum_{n=1}^{N} \sqrt{\frac{\varepsilon f_{s}}{\varepsilon_{m n}}} \frac{L}{d_{t o C H}^{2}}}
$$

where, $\varepsilon$ represents the optimal signals from the available signals.

The collision rate is reduced in the Integrated Signal Allocation Model with Effective Collision Resolution Model is performed as

$$
\begin{gathered}
\min \left\{\operatorname{Ch}_{\text {freq }}\left\{\left(\varepsilon_{n}^{L}\right)^{M} V_{n}^{L} \psi_{n}^{T h}\right\}\right\}= \\
C h_{\text {freq }}\left\{\begin{array}{l}
\left(\varepsilon_{n}^{L}\right)^{M}+\max \left\{\left(\operatorname{diag}\left(\hat{x}_{n}^{L}\right)\right)^{M} * C h_{f}\left(N L_{n}\right)^{T h}\right. \\
\left.* \operatorname{diag}\left(x_{m}^{L}\right)\right\}+\left(\varepsilon_{n}^{L}\right)
\end{array}\right\}
\end{gathered}
$$

The Normalized Mean Square Error (NMSE) of the collisions based on allotted signals are calculated as

$$
\begin{gathered}
N M S E=\frac{\varepsilon^{2}}{C h_{\text {frea }}} \frac{\sum_{i=1}^{L} \text { Strength }_{C H}\left\{\left(\varepsilon_{n}^{L}\right)^{T h}\right\}}{\sum_{i=1}^{N} B_{m}\left\{\left\|S_{n}+D L\right\|^{2}\right\}}+ \\
\frac{N L_{x y}\left(N_{s r c}-B_{m}\right)+\ell_{t}\left(2^{2 W}-1\right)}{S n\left(N L_{x y}+N_{y x}\right)+T h}
\end{gathered}
$$




\section{RESULTS}

The WSN topology is an essential factor to ensure that the actual settings in WSNs are represented comprehensively. Since the corresponding range of contact is approximately 80 $\mathrm{m}$ for $\alpha=4$, we set up $400 \mathrm{~m} * 400 \mathrm{~m}$, using different deployment schemes, with 50 sensors. The proposed Integrated Signal Allocation Model with Effective Collision Resolution Model (ICAM-ECR) model is compared with the existing Minimum Mean Square Error Combining Model (MMsECM). In our simulations, in networks with different settings, we test the proposed algorithms with parameters like Collision Rate, Collision Identification Rate, Collision Avoidance Levels, Node Labelling Time Levels, Packet Delivery Rate, Energy Consumption Levels and Throughput Levels.

A further main output parameter in WSNs is the collision rate. The WSNs' MAC layer protocol allows the frame to be retransmitted when collisions occur before the frame has been received successfully or exceeds the maximum number of retransmissions. This retransmission method is used to prevent endless transmissions through very poor links of consistency. The node drops the frame after it reaches the maximum number of retransmissions. This can cause the retransmission of a higher layer during which a new route is created. The loss of the frame, however, may be due to other sensor interference. The transmission of the top layer is also triggered in this case; however, this makes no sense, as congestion in WSNs is often within an area. During this method, however, the creation of a new transmission route costs tens or hundred times as many times as a regular MAC frame transmission. The Parameters used for the simulation are indicated in Table 1.

Table 1. Parameters used

\begin{tabular}{cc}
\hline Parameter & Value \\
\hline Number of subcarriers & 1024 \\
FFT size & 1024 \\
Type of modulation & $16,32,640 \mathrm{QAM}$ \\
Type of pilot insertion & Comb \\
Type of guard interval & Cyclic prefix \\
Length of guard interval & 256 \\
Channel model & UWA multipath channels \\
Noise model & Additive White Gaussian Noise \\
\hline
\end{tabular}

The proposed model reduces the collision rate for lossless data transmission. The Collision rate of the proposed and the traditional models are indicated in Figure 3.

The Collision Identification rate of the proposed and the existing models are indicated in Figure 4. The proposed model collision identification rate is high that improves the Quality of Service levels.

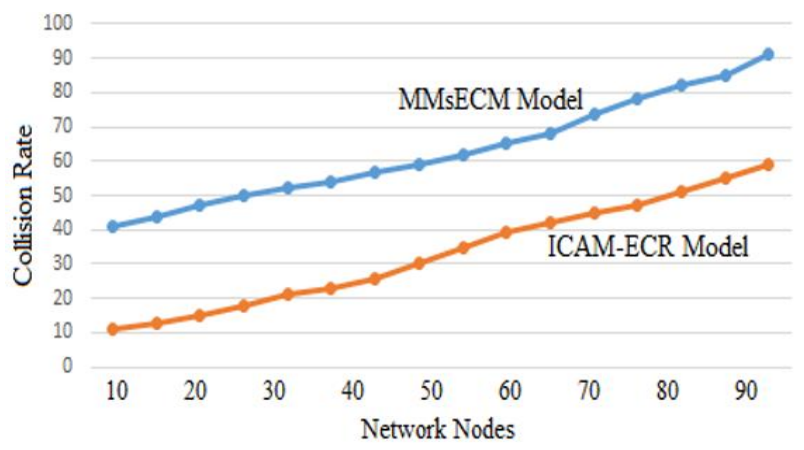

Figure 3. Collision rate
The proposed model effectively avoids the collisions by checking the nodes signal estimation and allocation levels. The collision avoidance levels of the proposed and existing models are represented in Figure 5.

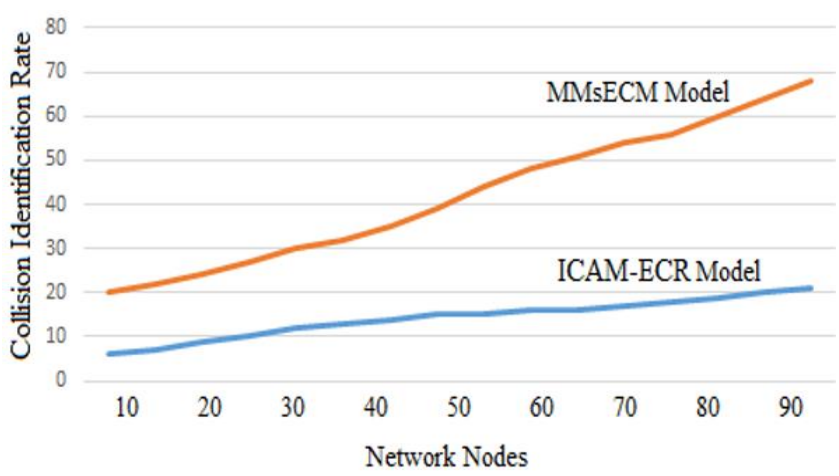

Figure 4. Collision identification rate

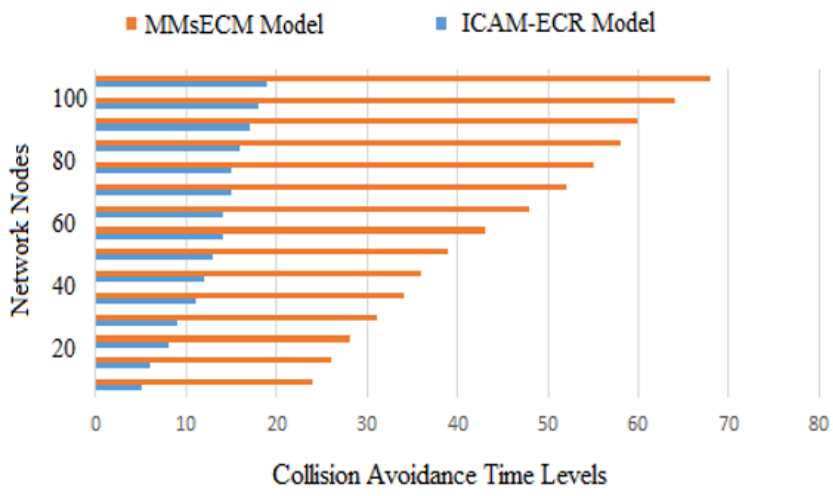

Figure 5. Collision avoidance time levels

The proposed model performs node labelling for checking which node is causing collisions. The proposed and existing model node labelling time levels are indicated in Figure 6.

The proposed model efficiently reduces the collisions during signal allocation thus the data can be transmitted without any loss. The packet delivery rates of the proposed and existing models are represented in Figure 7.

The energy consumption levels of the proposed model are less as the collisions and retransmissions are reduced. The energy consumption levels of the proposed and traditional models are indicated in Figure 8.

The overall throughput levels of the proposed and traditional method are indicated in Figure 9. The proposed model throughput levels are high when compared to existing ones.

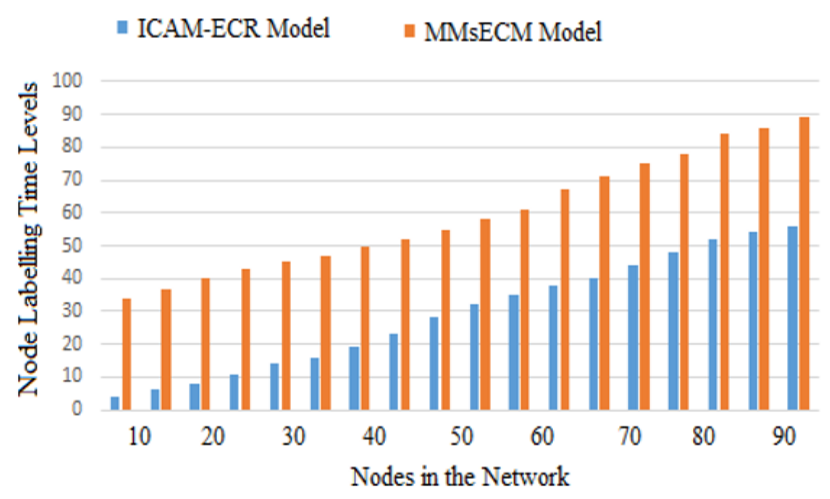

Figure 6. Node labelling time levels 


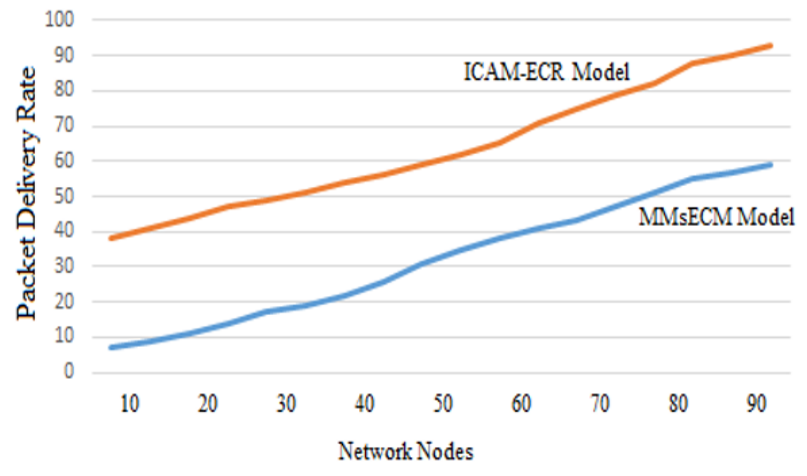

Figure 7. Packet delivery rate

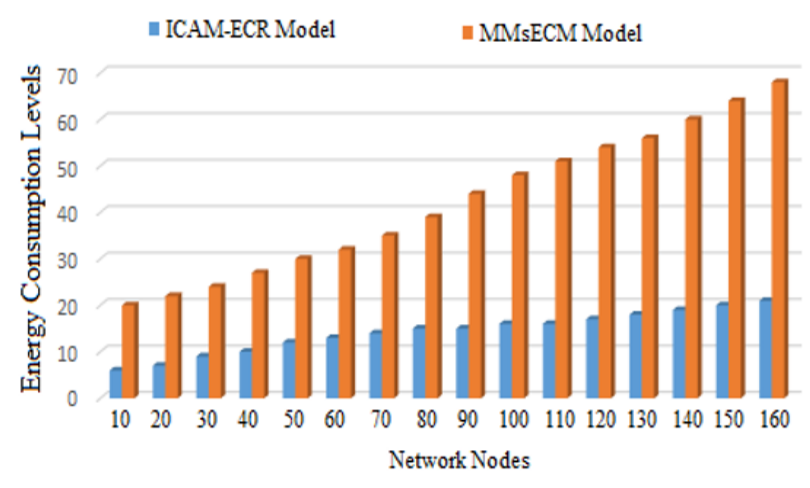

Figure 8. Energy consumption levels

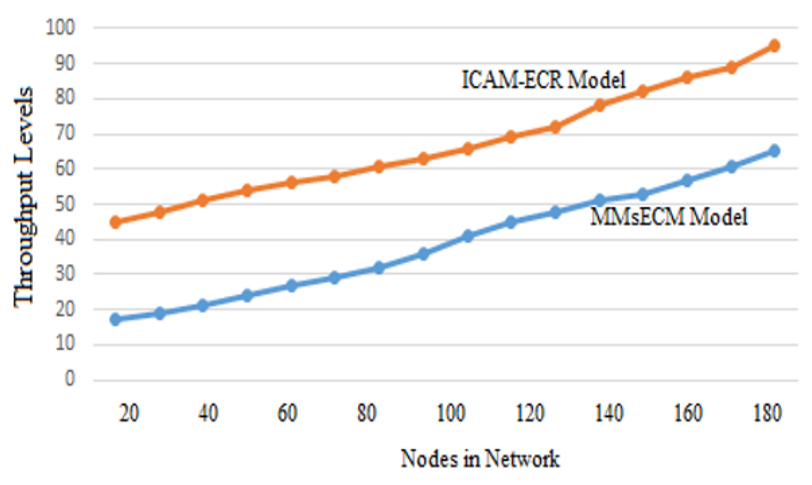

Figure 9. Throughput levels

\section{CONCLUSION}

Collisions can be a key cause of packet retransmission and hence an increase in energy usage within resource-constrained wireless sensor networks. The problem is resolved to some degree in traditional MAC-layer solutions and is relatively small. In this paper, an integrated signal allocation model is proposed for collision resolution and collision avoidance. In wireless IEEE 802.11 networks, collisions and interference in large and densely used WSNs affect significantly the throughput. The removal of interruption and collisions has been recognised as a research issue. This paper analysed the communication characteristics of WSNs and adequately suggested an integrated collision-oriented data transfer strategy. In order to compart the effectiveness of the proposed system, extensive simulations were carried out. The results of simulations show that with our proposed approach the rate of frame loss may be less than $2 \%$ for large-scale WSNs and that the mean network output may be increased efficiently to four times that of other signal access systems for WSNs. The proposed model reduces the collisions and avoids data retransmissions by $35 \%$ that improves the system performance and accuracy in data transmission. In future, back off models can be enhanced for collision resolution and the central authority in WSN can be selected for monitoring the data transmissions among the nodes in the network. The performance levels in reducing collisions can be still improved by signal frequency analysis continuously and using the remaining or idle time slots for better performance levels.

\section{REFERENCES}

[1] Lei, C., Bie, H., Fang, G., Mueck, M., Zhang, X. (2016). An efficient backoff algorithm based on the theory of confidence interval estimation. IEICE Transactions on Communications, E99.B(10): https://doi.org/10.1587/transcom.2015EBP3530

[2] Roy, N.R., Chandra, P. (2018). A note on optimum cluster estimation in leach protocol. IEEE Access, 6: 65690-65696. https://doi.org/10.1109/ACCESS.2018.2877704

[3] Sharma, D., Bhondekar, A.P. (2018). Traffic and energy aware routing for heterogeneous wireless sensor networks. IEEE Communications Letters, 22(8): 16081611. https://doi.org/10.1109/LCOMM.2018.2841911

[4] Kaur, T., Kumar, D. (2018). Particle swarm optimization-based unequal and fault tolerant clustering protocol for wireless sensor networks. IEEE Sensors Journal, 18(11): 4614-4622. https://doi.org/10.1109/JSEN.2018.2828099

[5] Behera, T.M., Samal, U.C., Mohapatra, S.K. (2018). Energy-efficient modified LEACH protocol for IoT application. IET Wireless Sensor Systems, 8(5): 223-228. https://doi.org/10.1049/iet-wss.2017.0099

[6] Obaidat, M.S., Misra, S. (2014). Principles of Wireless Sensor Networks. Cambridge University Press.

[7] Emad, A., Ion, M. (2018). New energy efficient multihop routing techniques for wireless sensor networks: static and dynamic techniques. Sensors, 18(6): 18631866. https://doi.org/10.3390/s18061863

[8] Jadoon, R.N., Zhou, W., Jadoon, W., Ahmed Khan, I. (2018). RARZ: Ring-zone based routing protocol for wireless sensor networks. Applied Sciences, 8(7): 1023. https://doi.org/10.3390/app8071023

[9] Tarhani, M., Kavian, Y.S., Siavoshi, S. (2014). SEECH: Scalable energy efficient clustering hierarchy protocol in wireless sensor networks. IEEE Sensors Journal, 14(11): 3944-3954. https://doi.org/10.1109/JSEN.2014.2358567

[10] Tanwar, S., Tyagi, S., Kumar, N., Obaidat, M.S. (2018). LA-MHR: Learning automata based multilevel heterogeneous routing for opportunistic shared spectrum access to enhance lifetime of WSN. IEEE Systems Journal, 13(1): 313-323. https://doi.org/10.1109/JSYST.2018.2818618

[11] Kim, D.S., Chung, Y.J. (2006). Self-organization routing protocol supporting mobile nodes for wireless sensor network. First International Multi-Symposiums on Computer and Computational Sciences (IMSCCS'06), Hangzhou, China, p2. 622-626. https://doi.org/10.1109/IMSCCS.2006.265

[12] Yan L., Pan W., Luo B., Li X., Liu J. (2011). Modified energy-efficient protocol for wireless sensor networks in the presence of distributed optical fiber senor link. IEEE 
Sensor Journal, 11(9): 1815-1819. https://doi.org/10.1109/JSEN.2010.2104317

[13] Priyadarshi, R., Singh, L., Singh, A., Thakur, A. (2018). SEEN: Stable energy efficient network for wireless sensor network. In 2018 5th International Conference on Signal Processing and Integrated Networks (SPIN), Noida, India, pp. 338-342. https://doi.org/10.1109/SPIN.2018.8474228

[14] Kang, J., Sohn, I., Lee, S.H. (2019). Enhanced messagepassing based LEACH protocol for wireless sensor $\begin{array}{llll}\text { networks. } & \text { Sensors, } & \text { 19(1): }\end{array}$ https://doi.org/10.3390/s19010075

[15] Cheng, H., Su, Z., Xiong, N., Xiao, Y. (2016). Energyefficient node scheduling algorithms for wireless sensor networks using Markov Random Field model. Information $\quad$ Sciences, 329: 461-477. https://doi.org/10.1016/j.ins.2015.09.039

[16] Hoang, D.C., Yadav, P., Kumar, R., Panda, S.K. (2013). Real-time implementation of a harmony search algorithm-based clustering protocol for energy-efficient wireless sensor networks. IEEE Transactions on Industrial Informatics, 10(1): 774-783. https://doi.org/10.1109/TII.2013.2273739

[17] Noh, Y., Lee, D. (2017). BCoPS: An energy-efficient routing protocol with coverage preservation. IET Communications, 11(12): 1933-1940. https://doi.org/10.1049/iet-com.2017.0038

[18] Miao, H., Xiao, X., Qi, B., Wang, K. (2015). Improvement and application of LEACH protocol based on genetic algorithm for WSN. 2015 IEEE 20th International Workshop on Computer Aided Modelling and Design of Communication Links and Networks (CAMAD), Guildford, UK, pp. 242-245. https://doi.org/10.1109/CAMAD.2015.7390517

[19] Singh, S.K., Kumar, P., Singh, J.P. (2017). A survey on successors of LEACH protocol. IEEE Access, 5: 42984328. https://doi.org/10.1109/ACCESS.2017.2666082

[20] Zhang, W., Wei, X., Han, G., Tan, X. (2018). An energyefficient ring cross-layer optimization algorithm for wireless sensor networks. IEEE Access, 6: 16588-16598. https://doi.org/10.1109/ACCESS.2018.2809663

[21] Zheng, H., Guo, W., Xiong, N. (2017). A kernel-based compressive sensing approach for mobile data gathering in wireless sensor network systems. IEEE Transactions on Systems, Man, and Cybernetics: Systems, 48(12):
2315-2327.

https://doi.org/10.1109/TSMC.2017.2734886

[22] Ahmad, I., Shah, K., Ullah, S. (2016). Military applications using wireless sensor networks: A survey. International Journal of Engineering Science and Computing, 6(6): 7039-7043. https://doi.org/10.4010/2016.1678

[23] Azzabi, T., Farhat, H., Sahli, N. (2017). A survey on wireless sensor networks security issues and military specificities. 2017 International Conference on Advanced Systems and Electric Technologies (IC_ASET), Hammamet, Tunisia, pp. 66-72. http://dx.doi.org/10.1109/ASET.2017.7983668

[24] Dubey, J.R., Bhavsar, A.R., Arolkar, H.A. (2017). Wireless sensor network based accident avoidance architecture for the fleet of long route vehicles. EAI Endorsed Transactions on Scalable Information Systems, 4(15): $\quad$ e3. https://doi.org/10.4108/eai.28-122017.153521

[25] Ehrlich, M., Wisniewski, L., Jasperneite, J. (2018). State of the art and future applications of industrial wireless sensor networks. Kommunikation und Bildverarbeitung in der Automation, pp. 28-39. https://doi.org/10.1007/978-3-662-55232-2_3

[26] Zhang, Y., Shen, Y., Wang, H., Yong, J., Jiang, X. (2015). On secure wireless communications for IoT under eavesdropper collusion. IEEE Transactions on Automation Science and Engineering, 13(3): 1281-1293. https://doi.org/10.1109/TASE.2015.2497663

[27] Khamespanah, E., Sirjani, M., Mechitov, K., Agha, G. (2018). Modeling and analyzing real-time wireless sensor and actuator networks using actors and model checking. International Journal on Software Tools for Technology Transfer, 20(5): 547-561. https://doi.org/10.1007/s10009-017-0480-3

[28] TelosB Datasheet. Available online: http://www.memsic.com/userfiles/files/Datasheets/WS N/telosb_datasheet.pdf, accessed on 3 March 2017.

[29] Pal, A., Nasipuri, A. (2014). Lifetime of asynchronous wireless sensor networks with multiple channels and power control. 2014 IEEE Wireless Communications and Networking Conference (WCNC), Istanbul, Turkey, pp. 2874-2879. https://doi.org/10.1109/WCNC.2014.6952905 